\title{
Mechanical Sorting Processing of Waste Material Before Composite Manufacturing - A Review
}

\author{
Ville Lahtela* and Timo Kärki \\ LUT University, School of Energy Systems, P.O. BOX 20, FI-53851 Lappeenranta, Finland
}

Received 29 December 2017; Accepted 20 November 2018

\begin{abstract}
This study reviews various mechanical sorting options in the field of solid waste. The interest towards the reuse of waste materials is growing due to the tightened regulation and increasing demand for recycling. The effective reutilization of waste material will be needed sorting technologies, which can be based on the different properties, such as density, electric conductivity, and other surface properties. Several technologies based on sorting were presented decades ago, but in the field of waste, several studies have been published recently that demonstrate to theme being is an essential issue currently. The review summarizes the potential mechanical sorting technologies for solid waste, which were discussed in accessible literature during the last decades. The different methods are included, based on the material properties such as, size, gravity, magnetism, surface tension, electric conductivity, or combinations thereof. The emphasis of technologies are targeted at the methods that are suitable for solid waste material, which can be reused as a raw material in the manufacturing of the products, such as composite production. This kind of operating model promote resource efficient production and contributing circular economy. It is concluded that the efficient sorting results of solid waste materials can be achieved by a combination of several technologies, but a lot of studies will be still needed in the future.
\end{abstract}

Keywords: Composite, recycling, separation, sorting, waste.

\section{Introduction}

With the world's increasing population, the generation of waste has risen considerably. For example in Malaysia, solid waste generation has increased by more than $90 \%$ for every 10 years due to population growth [1]. In addition to population growth, urbanization and industrialization have increased the quantity of municipal solid waste (MSW). For example, in China the total amount of MSW of a citizen per day increased from $0.50 \mathrm{~kg}$ to $0.98 \mathrm{~kg}$ between the years 1980 and 2006 [2]. Landfill and incineration have been the traditional and widely used methods for waste disposal, but they are not trouble-free and create environmental concerns, such as groundwater contamination, release of toxic gases, and odor problems. Incineration of certain materials may cause challenges for process equipment, like the pyrolysis of polyvinyl chloride (PVC) that forms acid gas (hydrochloric acid, $\mathrm{HCl}$ ) and causes corrosion in apparatus [3]. In addition, the increasing costs and decreasing areas for landfills increase the need to search for alternative options for waste disposal. However, landfill cost level can vary greatly depending on the waste type and the geographical region [4] but the development of cost has been significant in some places. For example in Finland, waste tax has risen to $75 \%$ between years 2011 and 2016 [5,6]. In addition to the issues mentioned above, concern for the sufficiency of certain raw materials is growing. For example, the European Commission has created a list of critical raw materials (CRMs) which have high importance and risk to the

\footnotetext{
*E-mail address: Ville.Lahtela@lut.fi

ISSN: $1791-2377$ @ 2018 Eastern Macedonia and Thrace Institute of Technology. All rights reserved. doi:10.25103/jestr.116.06
}

economy of European Union and their supply [7]. These facts have raised general interest in taking steps toward zero waste nation, which can be solved, inter alia, by recycling and reuse applications, and the sorting of waste stream has remarkable influence on the utilization of these. Therefore, contributing to circular economy is crucial work, not only from the viewpoint of resource and energy sustainability, but also environmentally.

Global governmental policies and the amendment of legislation are key drivers for the increased demand of research in re-materialization and circular economy. The terms waste and recycling are explained in a condensed form in Directive [8] as follows. Waste is any substance or object which is discarded, to be discarded, or is required to be discarded. Recycling is a recovery operation by which waste materials are reprocessed into products, materials, or substances. However, recycling does not include energy recovery and reprocessing into materials that are to be used as fuels of backfilling operations. Waste legislation and the policy based on the waste hierarchy have the following order of priority: prevention, preparing for reuse, recycling, other recovery, and disposal. The Member States of the European Union have set targets to increase the reuse or recycling of household waste to a minimum of overall $50 \%$ by weight by 2020. In addition, the corresponding rate in the same period, related to non-hazardous construction and demolition waste, is $70 \%$ [8]. In addition to previous decisions, the European Commission has adopted a legislative proposal, the aim of which is to increase circular economy in Europe. The proposal includes higher goals, such as increasing the recycling of municipal and packaging waste to $65 \%$ and $80 \%$, respectively, by $2030[9,10]$. In Finland, if waste is recovered for necessary structures or construction at 
landfills, it is exempt from waste tax, which is $70 €$ per ton of waste $[5,6]$.

Bezati et al. [11] have classified sorting into two categories: macrosorting and microsorting. Macrosorting deals with sorting as a whole, whereas microsorting takes place after the waste material has been chopped into pieces. This study is mainly focused on microsorting. Even though the price level of recycling infrastructure has raised doubts concerning its cost-effectiveness [12], the sorting systems have taken big steps during the last decades. However, the increasing material complexity and fast growing urbanization have increased the challenges in collection [13]. The importance of separation cannot be underestimated, as for example recent studies have shown that the share of sorting costs is $30-50 \%$ of the total system cost of packaging waste management [14].

Plastic is a small but very significant component of the waste stream, and it is an excellent example to demonstrate the evolution of waste management. Plastic is useful material to replace other materials in many industries due to its favorable features, such as functionality, hygiene, lightness, and cost. The world's total consumption of plastic has an average growth rate of 5-6\%, and this sharp rise and mass consumption produce a great amount of waste, which poses challenges for waste management [15]. The recycling rate of plastics can be increased remarkably in the future. For example, most post-consumer plastics will end up as energy or in disposal in Europe, but its reuse as a raw material in a composite structure, could be a certain option for waste plastic. The development of recycling has been expeditious in the last decades. For example, in the USA the quantity of plastics in municipal solid waste increased from 6.8 million tons to 31.75 million tons between the years 1980 and 2012. Correspondingly, the recycling rate increased from $0.3 \%$ to $8.8 \%$ in the same period [16]. The economic feasibility of recycling plastic depends on crude oil market prices and waste landfill costs. According to some calculations, the recycling of plastic waste is viable when the current oil market price is over US\$ 100 per barrel [17]. However, the cost of producing virgin materials may be less than the cost of collecting, cleaning, sorting and processing post-consumer plastics [18]. Plastic decomposes slowly, and incineration may be hazardous. Therefore, plastic waste should be recycled into reuse, but the problem is that different types of plastics have different chemical structures and they should not be mixed together. Selective separation of waste plastic is the weakest link in the plastic recycling industry [15]. Due to some quite similar properties of plastics, like density, which is presented in Tab. 1, the sorting can be challenging. The price of mixed plastics may also be negative in material recovery facilities [14]. For this reason, plastic sorting is one of the most commonly studied sorting systems. Sorted plastic can be utilized as raw material for composites, which material share can be a half of the total composition, for example. It must be remembered, however, that the recycling cycles are restricted because virgin plastic material contains small amounts of antioxidants, which are depleted during the processing phases, and this will cause degradation [19]. It has been found that polymer materials can be recycled five times without any significant changes [20]. According to the study of [21], the molecular weights decreased when the amount of recycling cycles of wood-plastic composites increased, but the mechanical tests showed no critical changes.
Table 1. Density of some commonly used plastics [22]

\begin{tabular}{cc}
\hline Plastic & Density $\left(\mathbf{g ~ c m}^{-3}\right)$ \\
\hline HDPE & $0.94-0.96$ \\
PP & $0.90-0.91$ \\
PET & $1.38-1.39$ \\
PVC & $1.20-1.42$ \\
PS & $1.05-1.07$ \\
ABS & $1.02-1.17$ \\
\hline
\end{tabular}

Another often-studied material in separation technology is the waste of electrical and electronic equipment (WEEE) [23], due to concern of fossil resource [24] and its valuable secondary resources [25]. WEEE poses also environmental challenges, as for example printed circuit boards (PCB) may contain toxic substances, such as brominated flameretardants (BFR), PVC, and heavy metals [26]. Waste PCBs can cause a rapidly growing disposal problem global all over the world [27]. The amount of WEEE has been increased with technological innovations and social development and at the same time, WEEE is one of the fastest growing waste stream globally [28]. The generation of electronics waste (ewaste) is rising three times faster than other forms of municipal waste [29]. The worldwide generation of WEEE has been estimated to be dozens of million tons per year, and most of it is landfilled or incinerated, which causes damage to the environment and human health due to the hazardous products contained in it [30]. The sorting of WEEE is challenging for recycling due to the many different components, but it is very rewarding and instructive at the same time, due to the valuable components that WEEE includes [31-33].

As shown above, reducing the adverse impacts of the management of waste is a global concern that needs solutions in the future. A certain solution can be the reuse of waste material in the composite production. In this paper, we present and discuss the available recently investigated treatment and sorting technologies, which could be possible to utilize with waste materials. The waste materials can be sorted by human sorters or robotic sorting, picking valuable objects from the waste stream. In this study, we do not focus specially on human or robotic methods, but we emphasize methods where separation is based on smaller particle sizes with large volumes and low cost, so-called mechanical separation. The aim of the study is to find technologies, which could be utilized as a part of composite production.

\section{Pre-processing before sorting technology}

Most technical sorting methods require pretreatment of the material. Generally, the first step in the sorting process involves size reduction of the material to a more suitable form. The size reduction and fragmentation are achieved with methods like milling, grinding or shredding. Also cleaning of the material can be performed before the actual separation if the process needs it. Sieving can also be a certain step before separation, concentrating specific materials into specific size fractions but in this study, it is presented as a sorting technology part "3.2 Screen sorting".

The pretreatment of material should not be underestimated. Excessive size reduction will increase the amount of dust, which may cause health issues and loss of valuable elements [34]. Particle size and shape have a significant impact on material processing, and therefore choosing the suitable sorting technique is essential [35]. Liberation is an important term in the recycling field, 
describing the distribution and connection of compounds in the recyclable streams [36]. Comminution is also the term used in this kind of context that describing the size and liberation distribution [37].

Size reduction can be performed for example by cutting, tearing or pulverizing. Hammer mill, impact mill and knife mill are examples of general size reduction equipment, just to mention a few. Hammer mills are widely used and their advantages are high productivity and flexibility [38]. In general, in hammer milling the material is fed into the mill device where it is ground or cut by impact of hammers against a wall. When the particles are fine enough, they are discharged to pass through a screen [39]. It has been reported that a hammer mill used less energy than a knife mill [40]. Plastics of a high degree of plasticity processed with a hammer mill may require subsequent processing with a cutter mill [41].

The impact mill device consists of several pins placed in a circular rotor. The material is fed into the mill with a vibrational feeder in the center of the mill, and the particles collide with the pins due to centrifugal forces. A circular sieve can be installed at the external part of the pins, but the mill can also operate without any sieve, in which case the particles are broken mainly due to impact loads. The ground material is collected in a circular container, which is located outside the pins and the optional sieve [42].

The knife shredder employs sharp and long knives to cut the material into small pieces. The knives are attached to a rotor of the shredder, and when the rotor rotates, the knives pass by an impact or cutting block at high speed. Hard materials are not suitable for a knife shredder, as they could damage the knives [43].

\section{Sorting}

There are several sorting methods, which utilize various features of materials, for example the hydrophobic/hydrophilic nature of the surface of the material or its electrical conductivity. For example, the sorting of plastic mixtures has been presented and investigated in several studies. Plastic sorting is quite understandable because different plastics have different physical characteristics, such as the melting point, hardness and density, and therefore mixed plastics have no significant practical value and they must be separated from each other.

\subsection{Manual sorting}

The simplest example of manual sorting can be found in modern households, where materials can be sorted into several categories. Manual sorting may be the easiest to organize, but its productivity is low [44] and it may not be economical [3]. According to one evaluation, machines have $30 \%$ better collection rate compared to household sorting for recycling [45]. Therefore, it does not seem to be a suitable method for a future technology in waste management. However, manual dismantling is a proper method in the case of WEEE when economic cost and quality assessment have been taken into consideration [46].

\subsection{Screen sorting}

Different screens and sieves can perform waste sorting. Screening is certainly the most general and oldest sorting method based on physical size. In spite of the long history, it is not thoroughly understood. Studies of screening have based on factors that describe the efficiency and kinetics of screening [47].

The trommel screen, also called trommelling, is a rotating cylindrical separation apparatus that separates particles according to size. The separation is based on a rotating perforated drum whose holes incrementally increase in size along the length of the cylinder. Depending on the design of the device, the smaller materials may drop out early in the process and the larger ones later. The advantages of the trommel screen are its simplicity in function and the relatively low need of capital, as well as low operating and maintenance costs. An important parameter in trommel screen separation is the rotational speed. The material should remain in the drum for at least two minutes $[47,48]$.

Ballistic separation is a similar method with the screen sorting, as its operation can be described as screening classifying and separation based on the specific weights of the waste fractions. Ballistic separation can sort waste in three fractions: fine, light and heavy. A ballistic separator includes a vibrating, inclined and perforated deck, and in action, heavy materials fall the lower level of the deck while lighter materials are transported upwards. Fine materials fall through the perforated bottom $[49,50]$. It has been found that ballistic separators can sort a mixed plastic waste stream successfully [51].

\subsection{Magnetic sorting}

Magnetic sorting is a method used for streams containing metal contaminants, and the magnetic parts are removed from the other materials magnetically. The method is based on the presumption that the magnetic force is greater than the gravitational force of the handled components. A magnetic sorting device is depicted in Fig. 1. The particles adhering to the surface of the feeding belt have magnetic force. If the gravity force of the particles is greater than the magnetic force, these particles will detach before the particles with high magnetic force. The particles are then collected separately. The feeding speed has been found to affect the sorting efficiency, which increases with increasing speed [52].

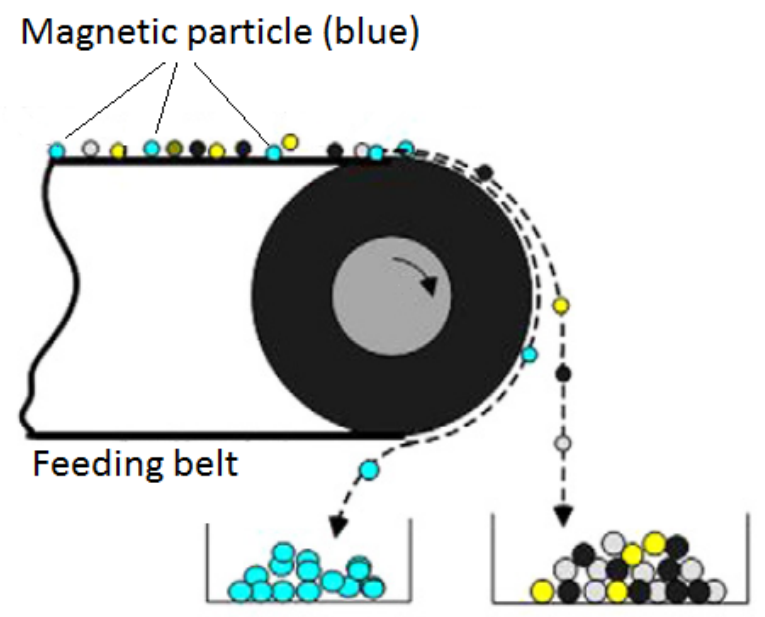

Fig. 1. Schematic diagram of magnetic separation. Adapted from Ruan and $\mathrm{Xu}[52]$

The magnetic density separation (MDS) method that utilizes the differences in the density of materials. It is performed by adding matter in the medium, for example magnetic iron oxide particles with the size of 10-20 nm. Therefore, the effective density of the liquid varies in the vertical direction due to the application of an artificial gravity. Typical steps in the MDS method are wetting, 
feeding, sorting and collecting. The MDS method is comparable to the conventional sink-float separation (presented below), but its advantages are a shorter process time and there have no limitations on color [53-55].

Bakker et al. [56] present a prototype of an inverse magnetic density separator (IMDS), achieving promising results with polyolefin fractions. The basic principle of IMDS is using magnetic liquids as a medium with congruent density with water, but in a gradient magnetic field, the force on the volume of the liquid is the sum of gravity and magnetic force. The IMDS separates the material feed into layers depending on the distance from the magnet, which depends on the density of the material and the apparent density of the liquid. For example, if the magnet is on top of the magnetic liquid, the magnetic liquid has lower apparent density than water. An example of this is shown in Fig. 2.

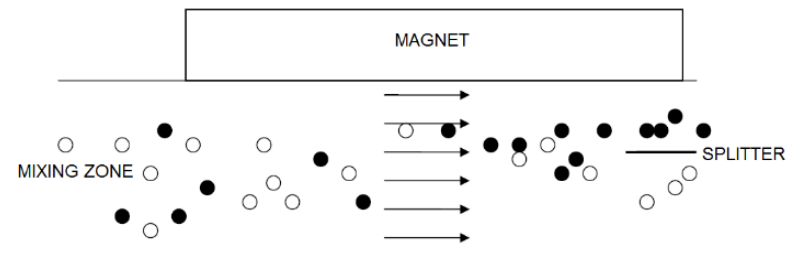

Fig. 2. The principle of IMDS for two different particles. Adapted from Bakker et al. [56]

\subsection{Gravity-based sorting}

The particles can be sorted according to their density differences. The most common ways for gravity sorting are centrifugal (cyclones), sink-float, and pneumatic sorting [57]. In addition, jig sorting is classified as a gravity concentration method [58]. Sorting based on different densities may be the most widely used material sorting process. It is a comparatively simple, easily automated and flexible operation, as well as high-capacity process, but on the other hand, it is rather slow and expensive [17,53].

\subsubsection{Centrifugal sorting}

Centrifugal sorting is also known as cyclone sorting, due to the apparatus of sorting. It is used extensively in mineral processing and in the recovery of metals from vehicle recycling. The operation of the cyclone is based on centrifugal forces. When the particles are fed into the cyclone, large and dense particles are forced against the wall and migrate downwards, while fine or low-density particles move upward to the overflow [59]. Common types of cyclones are conocylindrical cyclones and cylindrical cyclones. The approach of both types of cyclones are similar, and the main difference between the types is the operating angle. Cylindrical cyclones are operated vertically while conocylindrical cyclones are operated at about 30degree angle from the horizontal [41]. The cyclones are effective tools for sorting if the materials are finely ground but the weakness is that the tool requires typically more than one step [57].

Centrifugal sorters have been used to sort plastics with different densities and thicknesses, and several applications have been studied in recent years. The sorting of plastics by density is based on their hygroscopic properties, but the weakest point in this method is that the flakes tend to stick together in certain parameters [17,44]. Pascoe [60] found that the effective density sorting of plastics depended on the particle size and aspect ratio. Other properties, which have remarkable influence on the efficiency of plastic sorting, are the shearing effect, split ratio, and pressure drop.
Adjusting these parameters will increase to the sorting efficiency [44].

Hydrocyclones are also often mentioned in the sorting field in the connection of liquid components in the separation material, for example slurry. Hydrocyclones can be used to sort particles with different sedimentation velocities [59]. Hydrocyclones have been found to be problematic in the sorting of low-density plastics due to the liquid [60].

\subsubsection{Sink-float sorting}

The sink-float sorting method sorts materials depending on their different densities in the process medium. The method is quite simple, but it may also be challenging if the densities of the materials are almost similar to each other. The intermediate density of the process medium must be between that of the sorted particles. For example, plain water is a suitable medium for sorting if the densities of the sorted materials are higher and lower than $1 \mathrm{~g} / \mathrm{cm}^{3}$. If the densities are something else, innovative solutions for the process medium need to be applied $[55,57]$.

The sink-float method is a suitable application for plastic sorting as well. As mentioned above, PVC plastic should be sorted to a different category from other particles due to its hazardous properties, and as can be seen in Tab. 1, it has heavy density $\left(1.20-1.42 \mathrm{~g} / \mathrm{cm}^{3}\right)$ and thus sink-float separation seems to be a suitable method for it. Truc and Lee [23] state that the sorting of polyethylene (PE) or polypropylene (PP) is easy in the sink-float due to their lower density compared to water. Similar density of the sorted materials may be challenging in the sorting, and water disposal may cause concerns in some treatment phases [61]. Sorting by the sink-float method may require several treatment steps, which may be problematic [56]. The sinkfloat method is sometimes combined with flotation (presented below), and several recycling processes start by sink-float sorting, and the heavier plastics will be sorted by froth floatation afterwards $[15,55]$. For example, Dodbiba et al. [62] have studied this treatment combination for plastics.

The jigging sorting method has a similar function as the sink-float method. It is a gravity concentration method based on a pulsating bed. A mixture of solid and water is placed in a perforated vessel and vertical currents of water are employed to form a pulsating bed. The heavier particles of the mixture sag down and stay inside the jigging cell, while the lighter particles rise up and overflow. The density and the size and shape of the particles are important parameters affecting the results [58].

\subsubsection{Air-gravity based sorting}

In addition to centrifugal and sink-float sorting, there are sorting methods based on a combination of air and gravity. Examples of these are pneumatic sorting and an air table utilizing air as power in the sorting. The airflows can be adjusted by the wind speed of the fan [52]. Pneumatic sorting is used to sort light or fine particles from the stream of the material process [41], but it may require a large industrial plant and a rather high cost [57].

Air tabling is dry gravity sorting that requires different densities for the sorted particles. A wider density range improves the results and according to an estimation, 450 $\mathrm{kg} / \mathrm{m}^{3}$ is the minimum difference between the densities. The dry state in the sorting phase increases the environmental benefits of the method, due to the lack of water and chemicals. For effective sorting, the minimum particle should be greater than $1.59 \mathrm{~mm}$ according to the study of Dodbiba et al. [63]. Other advantages are low capital and 
operational costs. An air table apparatus consists of a porous deck powered by an eccentric drive to impart longitudinal vibration. The porous deck is tilted and below the deck is located an electric fan that generates an upward airflow. Collecting bins are located at the end of the deck where the fractions are sorted according to density. One of the most important operating parameters of the air table is air velocity [64]. The principle of an air table device is presented in Fig. 3.

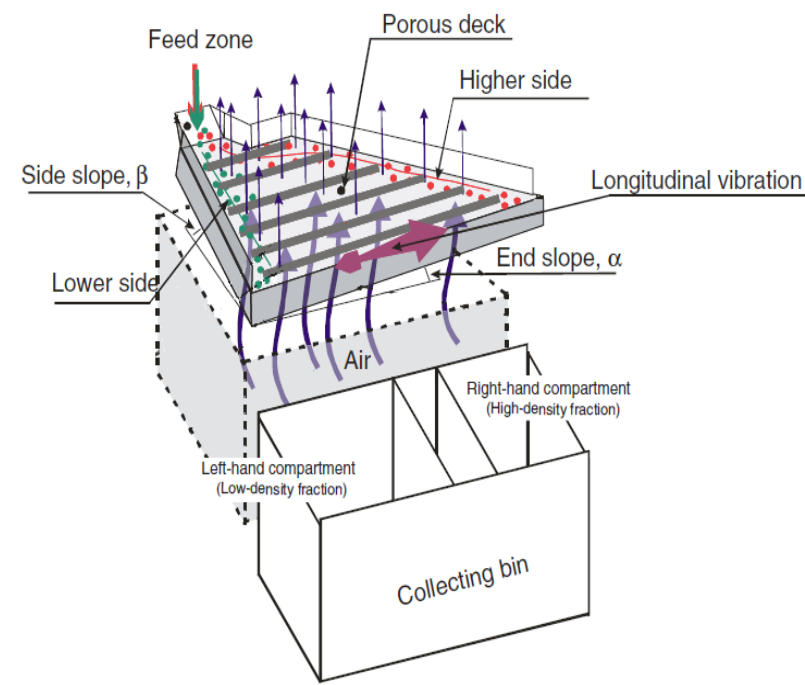

Fig. 3. The principle of an air table apparatus. Adapted from Dodbiba et al. [63]

A similar method with the previous one is the wet shaking table, also known as a water table, which includes also water motion in the inclined rectangular deck. The particles feed is introduced from the corner of the deck and they move diagonally across the deck so that the heavier particles will travel longitudinally and the lighter particles will travel with the wash water flow. The sorting is based on the combination of the asymmetrical motion of the deck, water movement and turbulence flow between the riffles. The performance of the apparatus depends on the number of parameters, and the design of the table has a significant effect on the function. Wet shaking tables have been used in mineral processing and coal cleaning. Its advantages are, inter alia, that it does not require high range density differences between the materials and its operating costs are low [65].

\subsection{Flotation}

Flotation was originally developed for ore sorting, and the principle behind it is based on the surface tension and hydrophobic properties of surfaces that have a contact with bubbles. The gaseous bubbles come to a contact with the hydrophobic particles and the particles are carried on the top of the flotation apparatus, from where it can be removed $[15,66,67]$. The principle of flotation sorting is presented in Fig. 4.

For the attachment of bubbles to be sufficient in the flotation sorting process, different wettability abilities between the particles are required. If the wettability abilities are not dissimilar enough, some surface treatment for the material may be necessary. Examples of this kind of methods are, inter alia, plasma exposure, ozone treatment, and high energy irradiations with electron, gamma, or UV radiation. In addition, treatments such as wet oxidation, flame treatment and boiling treatment have been mentioned to change the surface properties. Alterations in the material surface can be also achieved through the action of reagents, such as lignosulfonate, tannic acid, methylcellulose, and quebracho, for example. The pretreatment methods have also some of disadvantages. The equipment for high energy irradiation may be expensive and not safe for the environment, whereas chemical treatment needs time, facilities, and treatment for wastewater. Overall, surface modification depends on the solvent quality, reaction time, and temperature [67-70].

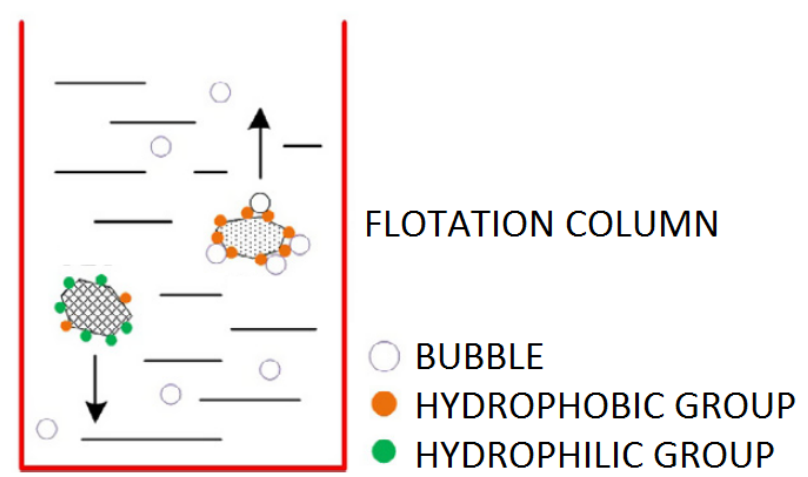

Fig. 4. Mechanism of flotation treatment. Adapted from Wang et al. [108]

Sorting based on the flotation technique is a useful and promising method to sort different types of plastics. However, understanding the chemical structure of the material has a crucial role. For example, PVC, which includes chloride as a hazardous feature, has been sorted from the plastic stream with the flotation technique in many studies. Mallampati et al. [70] treated various plastics with calcium, and the wettability of PVC was clearly altered. Calcium treatment decreases the chlorine concentration and increases the oxygen concentration of $\mathrm{PVC}$, which contributes to changes in the surface morphology and roughness of PVC. Pongstabodee et al. [22] found that the use of calcium chloride increased the wettability of plastic due to the calcium ions that acted as a bridge between the plastic surface and the wetting agent. Wang et al. [69] found that surface modification with a potassium permanganate solution changed the PVC surface selectively, while polyethylene terephthalate (PET) was insensitive to surface treatment under the experimental conditions. The mechanism of surface modification may have been due to oxidization reactions occurred on the PVC surface. Wang et al. [69] studied the separation of PET from waste by froth flotation. The weakest chemical connection in the PET chain is the ester link, and it is destroyed by a sodium hydroxide $(\mathrm{NaOH})$ solution, turning the surface more hydrophilic. Overall, plastic sorting based on the flotation technique is a relatively new method, and its favorable features are, inter alia, cost-effectively and high sorting efficiency [23,66-68]. The kinetic behavior of plastic has a crucial role on the flotation results [71].

The particle size has a clear influence on the floatation behavior. When plastic flotation is compared with the traditional ore flotation, the significance of particle size comes clearly out. The magnitude of plastic flotation is clearly larger than that of ore flotation, and therefore a single bubble is capable of carrying several solid ore particles, while one plastic particle needs numerous bubbles in the 
flotation method. It is estimated that the plastic particle size may be 2.0-6.0 $\mathrm{mm}$, depending on the flotation device [15].

\subsection{Electric conductivity-based sorting}

Electrostatic devices are used for sorting metal from plastics $[24,72]$. There are a few typical separation techniques, which are based on electric conductivity, such as eddy current, triboelectric, and corona electrostatic sorting. It has been stated that electrostatic sorting may be economically problematic if the throughput is high [56].

\subsubsection{Eddy current sorting}

Eddy current sorters (ECS) are some of the most significant developments in the recycling industry, the operability of which is based on the use of rare earth permanent magnets [35]. The patent for an eddy current sorter was applied in 1889 , but successful development was done in the late twentieth century. The principle of ECS technology is based on Faraday's law of electromagnetic induction and the alternating magnetic field [73]. As many other sorting technologies, also ECS has been employed in mineral processing in the past years [74]. It is a widely used sorting system for the recycling of non-ferrous metals from solid wastes [72], and it has been an effective sorting technology in recycling light metals from end-of-life vehicles [75]. The ECS systems include specific sorter types, like the horizontal drum eddy current sorter (HDECS) and the single disc eddy current sorter (SDECS) [76]. The diagram of ECS is presented in Fig. 5.

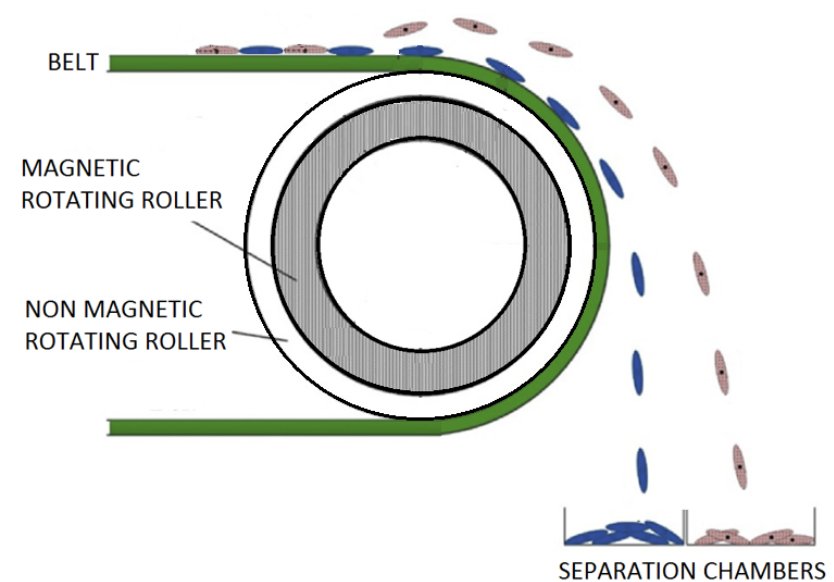

Fig. 5. Diagram of the eddy current separator. Adapted from Li et al. [77]

The main operating part in the ECS system is a rotating drum that is covered with alternating magnets. Various concepts have been tried, but the rotary drum type is the most widely used ECS process. The fluctuating field of the drum induces eddy current forces into conductive particles that move close to the drum and conducting particles are ejected by magnetic force. The rotor design has an actual role in the separation performance. The eddy current forces, together with gravity, centrifugal force, and other mechanical forces will cause a distinct trajectory for the particles [72,77].

The detachment angle is one of the parameters in eddy current sorting. The detachment angle is in connection with the sorting distance, which decreases with the growing of detachment angle. It has been found that a detachment angle of over seven degree with low enough feeding speed leads to complete sorting. Too high feeding speed may influence the sorting unfavorably. A bigger detachment angle means that the intensity of the magnetic field is weaker [74].
ECS has been used to recover particles from crushed ewaste, such as sorting of aluminum from the plastic in the recycling of cartridges and plastic sorting from printed circuit boards [75,77]. The sorting of plastic from printed circuit boards has achieved over 95\% efficiency [77], but in the case of toner cartridge recycling, effective sorting needs repeating and even manual sorting [75]. Great differences in size, shape and purity between the crushed e-waste particles decreases the sorting efficiency [74]. The sorting efficiency of ECS is the best when the particle size is over $5 \mathrm{~mm}$, or even $10 \mathrm{~mm}$ [35], and the moisture content should be 10$15 \%$ [72]. The feeding speed and the rotation speed of the magnetic field have also an influence on the results. It has been found that the rotation speed of the magnetic field must be sufficiently high for effective sorting [52].

The advantages of ECS are its low cost and high efficiency. In the future, ECS can sort smaller particles and extend their applications. It may replace some other sorting systems, like corona-electrostatic and air current sorting in ewaste recovery. However, the ECS process needs a lot of knowledge, an example of which are the various equations in the study of [74], and this may be challenging and laborious.

\subsubsection{Triboelectrostatic sorting}

Triboelectrostatic sorting is a method that sorts particles based on charging mechanisms, more exactly tribo series of materials through an electric field. The method is illustrated in Fig. 6. The contact of two materials that have different surface properties, create a charge between them, which is called the tribocharging phenomenon, also known as "contact electrification" or "frictional electrification". Sorting utilizes this tribocharging phenomenon, where the materials have different charged polarities. Depending on the charge of the material, it moves toward the positive or negative electrode, and the materials are collected. In triboelectrostatic sorting, the importance of selective charging and optimum charge density of the materials are highlighted, and therefore it is an inexpensive and efficient sorting method $[78,79]$.

Triboelectrostatic sorting needs some pretreatment of the material before the actual sorting. Firstly, the material must be crushed to optimum size, and sieves can be needed for the classification. The particle size has a significant influence on the efficiency of the sorting because, tribocharging happens on the surfaces of particles, for example as deep as $30 \mathrm{~nm}$ for polymers. A large surface area may decrease the electric field force, so the particle size for sorting has a maximum limit. On the other hand, too small particle size influences the process negatively, increasing the cost in the milling step and causing collisions of the same kind of particles, for example. Because the materials may get charged due to the pre-treatment methods, a discharger must neutralize them. Washing and drying might be necessary treatments before sorting. The commonly used tribochargers include following elements: cyclone, vibrating feeder, fluidized bed, inclined rotating drum, honeycomb, and spiral tube charger [61,79].

The charger of the material is an important factor in the efficiency of the tribocharging process. It is desirable that the charger is made from the same material as some of the particles in the mixture that passes through the charger. Charging can be performed for the pretreated materials by two mechanisms, the so-called solid single-phase mechanism and gas-solid two-phase mechanism. The solid single-phase mechanism means a charger type of e.g. a rotating tube, rotary blades, and vibrating devices. The 
charger types of gas-solid two-phase mechanisms are, inter alia, fluidized-bed, cyclone, and propeller-type chargers. In the rotating tube, the particles are fed into a slightly horizontally inclined rotating tube and the particles pass though the tube due to the force of gravity. The charge magnitude can be improved by adjusting the incline angle or the length of the tube. The tube may contain ribs, which improve the degree of mixing. The advantages of the rotating tube are, inter alia, their mechanical simplicity and modest power requirements. The disadvantages of the rotating tube are a low collision frequency between the particles, and other mechanisms may have higher charging efficiency. The rotating tube has been found to be feasible for some plastic mixtures. In the process of the fluidized bed, air turbulence is generated in the chamber and the particles are carried upwards with a gas flow, which enables the particles to be affected by the forces of gravity, air resistance, and air dynamic pressure, causing contacts between the particles and with the wall material. The layout of the fluidized bed can be varied, but the main idea is that the particles can rub against each other and get enough charge in the electric field [79]. A high-applied voltage and small inter electrode distance contribute to the results of separation [80].

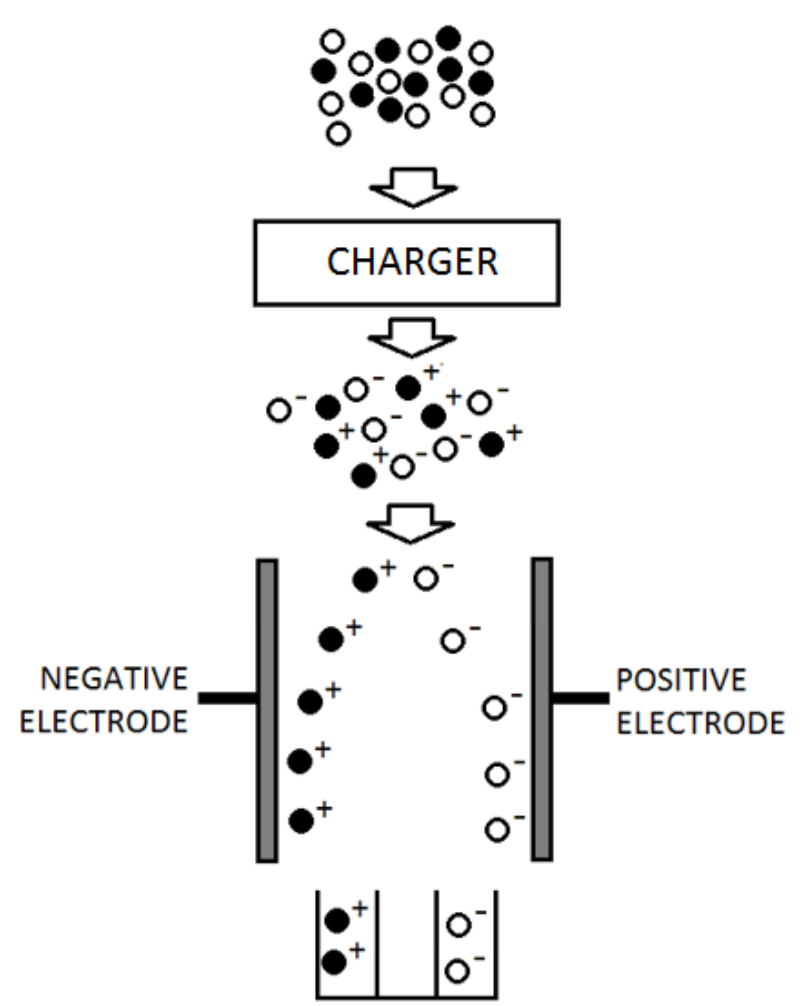

Fig. 6. The main principle of triboelectrostatic separation. Adapted from Lee and Shin [81]

Different plastics have different triboelectricities, as shown in Tab. 2. The triboelectrostatic system has been shown to be a potential method for plastic sorting and removing certain plastic types from the mixed waste stream $[35,81]$. For example, three types of shredded plastics were sorted in the two-stage process by triboelectrostatic system in the study of Park et al. [61]. The particles were neutralized after shredding, and afterwards they were fed into a cyclone charger with flowing air, where two particles were positively charged and some particles negatively, which were removed in the first stage. In the second stage, the two remaining particles (positively charged) were neutralized with the discharger and fed into the cyclone charger with flowing air. Due to the new charger, the particles had dissimilar charge. Hence, the charged materials turned to positive or negative electrodes (ibid.). According to $\mathrm{Wu}$ et al. [79], this is an especially suitable method the crushed or granular plastic waste, and many studies of triboelectrostatic sorting for plastics have been done. One example of the utilization of the triboelectrostatic method in plastic sorting is the removing of hazardous PVC from other plastics. PVC has a negative charge and other plastics have a positive charge, and thus PVC can be removed by electrostatic deflection and collected on a positive electrode [79]. The study of Bendimerad et al. [82] showed that a simple triboelectric sorter was effective in producing pure materials between PVC and PE wastes. However, it has been reported that plastics do not have a universal triboelectric charge, which can affect the results, as well as the additives in commercial plastics [79].

Table 2. Triboelectric series of plastics $[66,78]$

\begin{tabular}{|c|c|c|c|c|c|c|c|c|c|c|c|}
\hline \multirow{2}{*}{$\begin{array}{l}\text { Refe } \\
\text { renc } \\
\text { es }\end{array}$} & \multicolumn{3}{|c|}{$\underset{(+)}{\leftarrow}$ positive } & & \multicolumn{3}{|c|}{ Charging } & & \multicolumn{3}{|c|}{$\begin{array}{c}(-) \\
\text { negative } \rightarrow\end{array}$} \\
\hline & & $\mathrm{P}$ & A & P & $\mathrm{P}$ & $P$ & $\mathrm{H}$ & $\mathrm{L}$ & $\mathrm{P}$ & $\mathrm{P}$ & P \\
\hline et & $P$ & M & B & C & $\mathrm{S}$ & $\mathrm{E}$ & D & D & $P$ & V & $\mathrm{T}$ \\
\hline al. & U & M & $\mathrm{S}$ & & & $\mathrm{T}$ & $P$ & $\mathrm{P}$ & & C & $\mathrm{F}$ \\
\hline & $\mathrm{R}$ & A & & & & & E & E & & & $E$ \\
\hline Shen & & PO & $P$ & $\mathrm{P}$ & A & $\mathrm{P}$ & $P$ & P & $P$ & P & $\mathrm{P}$ \\
\hline $\begin{array}{ll}t & e t\end{array}$ & $\mathrm{P}$ & M & C & A & B & $\mathrm{S}$ & E & P & E & V & V \\
\hline al. & U & & & & S & & & & $\mathrm{T}$ & $\mathrm{C}$ & D \\
\hline & $\mathrm{R}$ & & & & & & & & & & F \\
\hline
\end{tabular}

The favorable features of triboelectrostatic sorting are high efficiency, low cost, wide range of particle size, and no concern of water disposal or secondary pollution [79]. Cui and Forssberg [35] have noted that the particle size in triboelectrostatic sorting should be below $5 \mathrm{~mm}$, but in plastic sorting, the range of particle size has been mentioned to be wider (1-13 mm). Triboelectrostatic sorting is cheaper and more efficient compared to the conventional sorting methods, but it also includes some disadvantages, such as sensitivity to the change of ambient humidity. In addition, triboelectrostatic sorting cannot process mixtures of three or more kinds of material at one time. If sorting is needed for several materials, a combination with other sorting methods is possible [79].

\subsubsection{Corona electrostatic sorting}

Corona electrostatic sorting (CES) uses corona charging to sort materials based on the differences in the density and electric conductivity of the materials. An excellent case for corona electrostatic sorting is separation between metals and non-metal materials. The sorting efficiency is based on the electrode system, rotor speed, moisture content, and particle size $[26,35]$. A representation of a corona electrostatic sorter is shown in Fig. 7, with a one-roll CES. A two-roll CES has also been investigated and compared with a one-roll CES. It was noted that the two-roll CES improved the stability and reliability of the sorting system, and it is a suitable system when the variation range of the particle size is large. Obviously, the two-roll CES increases the production capacity, and it decreases the amount of middling products $[26,83]$. Parallel system with CES is plate-type electrostatic sorters where the rotating cylinder has been replaced by (Sshaped) metallic plate $[84,85]$. 


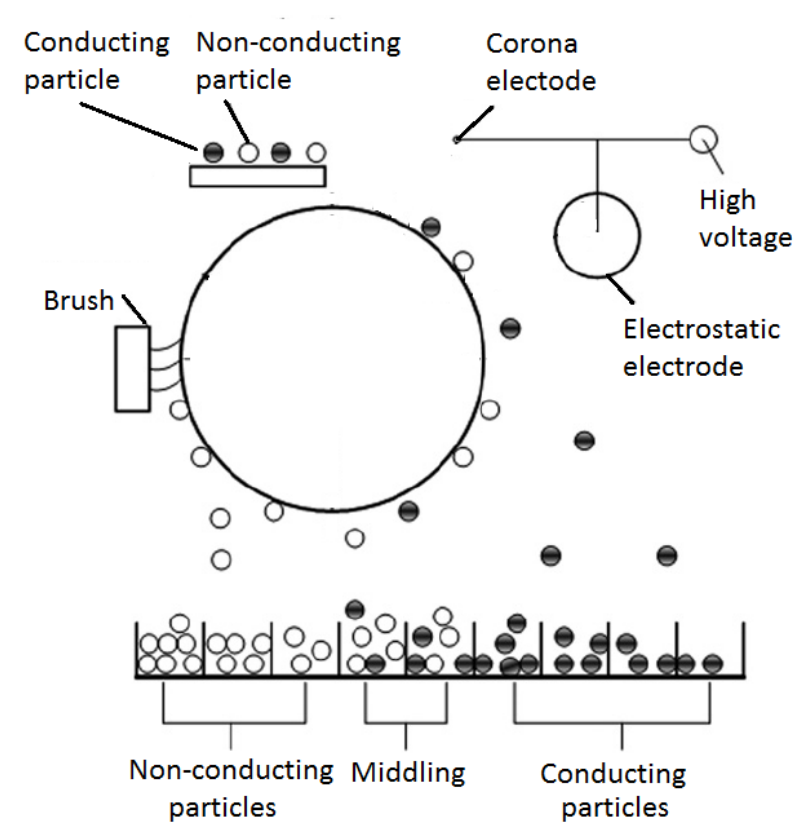

Fig. 7. A corona electrostatic separator. Adapted from Wu et al. [83]

The sorting based on an electrostatic field that is generated by a corona electrode and an additional electrode. The electrodes create forces that act differently between the materials. The corona electrode induces so called ion bombardment, while the additional electrode induces electrostatic induction, and so the electric conductive materials (metal) discharge rapidly. In turn, the charged materials are adhered to the rotating roll and move with it, and fall in the holding tank [86].

CES is an effective method for sorting small particles. The workable particle size of corona electrostatic sorting ranges between 0.1-5 $\mathrm{mm}$ [35], but particle sizes between 0.6 and $1.2 \mathrm{~mm}$ are the most feasible for industrial applications [86]. Very fine-grained particle size may cause problems, because they may have greater van der Waals force compared to the gravity force, which causes aggregation [87]. The particle shape has also an influence on the efficiency of CES [26]. Bedeković et al. [88] found that sorting is dependent on the electrode potential and rotor speed. The recovery increased with increasing electrode potential, while the increasing rotor speed decreased recovery. The rotor speed is more critical in the case of small particles than big particles [89]. It has been found that the capacity of the CES decreases with the decreasing size of samples $[86,88]$.

CES has been studied in the mineral-processing industry and it is used for recovering metals and plastic from PCBs $[83,86]$. It is also a useful method in waste treatment [89]. The advantages of the CES method are high productivity and low energy cost. It also offers no environmental challenges, like wastewater or gas during the process. The CES method includes many parameters, and some numerical simulating software may be useful for optimize the separating efficiency of CES [90]. It can also save human costs and help the new technology to be more easily applied in the industry [87].

\subsection{Image-based and optical sorting}

Imaging is becoming increasingly interesting for industrial applications [91]. Limiting factors for image-based automated sorting, such as X-ray detection, near infrared (NIR) spectroscopy, or optical sorting are their high cost, and the fact that their feed must be quite homogenous regarding for example size and shape $[88,92]$.

\subsubsection{NIR sensor based sorting}

Sensor based sorting utilizes the physical-chemical properties of the studied materials, such as density, electrical conductivity, magnetic susceptibility, or surface and material properties, as in Near Infrared (NIR) sensor based sorting [93]. The first application of NIR spectroscopy was tested in the USA in the 1950s. Today, several industrial applications utilize NIR Instruments, recording transmittance or reflectance for a set of wavelengths [91]. The principle of NIR-based sorting acts as follows. Unsorted materials move on a conveyor belt and are detected by a sensor. According to the measurements of the sensor, jets of compressed air linked to the sensors sort the materials. The capacity of the method depends on the bulk density and grain size of the input fraction [93]. NIR spectroscopy has been used to sort treated and untreated waste wood from each other, because the method can identify the type of inorganic preservatives [94].

As noted above, NIR spectroscopy is widely used industrially, due to its favorable features. Its advantages are e.g. a low environmental impact as well as rapid and reliable identification, but there are also some disadvantages, such as size and color limitations. It has been found that too big or too small objects are not favorable for the NIR method. Serranti et al. [55] state that the particle size should be bigger than $5 \mathrm{~cm}$. If the materials include small contaminants, it may require some other combination technology to achieve beneficial results. It has been noted in several studies that the technology is unable to sort dark materials and materials, which have close to similar properties with each other [53,56,61,93,95,96]. Labels, paint, and coating of the treated materials have an influence on the results [55]. According to the studies of Lofti et al. [96] and Vegas et al. [93], the representative purchase cost of NIR sorting system is hundreds of thousands of euros, depending on the capacity, but also higher purchasing costs for NIR sorters have been mentioned [97].

\subsubsection{X-ray sorting}

$\mathrm{X}$-ray fluorescence (XRF) works at the atomic level, based on the wavelength of X-rays that identify the elements [98]. Even though the use of X-rays is limited [99], XRF system has been used for the analysis of pressure treated wood $[100,101]$ and the identification of flame-retardants in WEEE [102]. In addition, plastic identification based on an $\mathrm{X}$-ray fluorescence detection system has been used together with tracers. Depending on the parameters used, it is a suitable method for high-speed identification of plastic material, and the dark color of plastic is not a problem $[11,99]$.

$\mathrm{XRF}$ is a multi-elemental non-destructive technology for the identification of different properties. Its advantages are, among others, that it does not require major prior preparation and a particular speed. Therefore, it is suitable for online applications [101]. The disadvantages of the XRF method are overheating due to high ambient temperature and heat production by the X-ray, coupled with poor cooling of the XRF chamber [100].

\subsubsection{Other optical sorting methods}

Hyperspectral imaging (HSI) utilizes the spatial and spectral information of the investigated object, characterized by two spatial dimensions and one spectral dimension. HSI is a non- 
destructive technology that has rapidly emerged in several industrial sectors, may be because it can analyze several physical and chemical characteristics of a sample at minor cost [103]. HSI technology is based on the utilization of an integrated hardware and software architecture that can capture and handle spectra according to a pre-defined alignment [95].

\section{8 (Other) test drives for sorting}

In addition to the abovementioned sorting technologies, it has been developed various other methods to perform sorting that may be potential in the future. One of these methods is the Laser-Induced Breakdown Spectroscopy (LIBS) technique that is based on the analysis of the atomic emission lines on the sample surface, generated by highenergy laser radiation. The strength of LIBS is exploiting a real-time process [96,104]. Lupo et al. [57] have tested a hydraulic sorter that may be a potential apparatus in the field of waste recycling in the future. The hydraulic sorter consists of semi-cylindrical transparent tubes that are welded in a plane, forming a channel where the waste flow moves along the lateral direction with water as the working fluid. The transparent tubes allow optical access during the process.

\section{Analysis and discussion}

Most of the studies dealing with waste material treatment and sorting technologies are quite recent, which indicates the importance of the topic at present. Every sorting method includes some positive and some negative properties, and some of them are presented in Tab. 3. The common property for every method in the table is non-destructive measurement, which can be classified as a positive property. The positive and negative properties of a method and their effects may cause challenges, and therefore they must be evaluated case by case.

Table 3. Positive and negative key points of various separation methods

\begin{tabular}{|c|c|c|}
\hline $\begin{array}{l}\text { Separation } \\
\text { method }\end{array}$ & $\begin{array}{l}\text { Strengths \& } \\
\text { Opportunities }\end{array}$ & $\begin{array}{l}\text { Weaknesses \& } \\
\text { Threats }\end{array}$ \\
\hline Manual & $\begin{array}{l}\text { Simple } \\
\text { Easy to organize } \\
\text { Several fractions possible }\end{array}$ & $\begin{array}{l}\text { Low productivity } \\
\text { Labor cost } \rightarrow \text { Economy }\end{array}$ \\
\hline Screen & $\begin{array}{l}\text { Simple } \\
\text { Low costs } \\
\text { Adjustment possibility }\end{array}$ & Depending on the size only \\
\hline Magnetic (dry) & $\begin{array}{l}\text { Efficient } \\
\text { Reliable method }\end{array}$ & Need for magnetism \\
\hline Magnetic (liquid) & $\begin{array}{l}\text { Short process time } \\
\text { No size limitation }\end{array}$ & Need for medium/agent \\
\hline Gravity-based (air)* & $\begin{array}{l}\text { Simple } \\
\text { Easily automated } \\
\text { Flexible }\end{array}$ & $\begin{array}{l}\text { Wide density differences, } \\
\text { Dust } \\
\text { Slow process }\end{array}$ \\
\hline Gravity-based (sink-float) & $\begin{array}{l}\text { Simple technology } \\
\text { Cleaning at the same time }\end{array}$ & $\begin{array}{l}\text { Weed for medium/agent } \\
\text { Water disposal } \\
\text { Density difference }\end{array}$ \\
\hline Flotation & Cleaning at the same time & $\begin{array}{l}\text { May need pretreatment } \\
\text { Size effects }\end{array}$ \\
\hline Eddy current & $\begin{array}{l}\text { Efficient } \\
\text { Low cost }\end{array}$ & $\begin{array}{l}\text { Moisture and size affect the result } \\
\text { Repetition or pretreatment } \\
\text { Need for knowhow }\end{array}$ \\
\hline Triboelectrostatic & $\begin{array}{l}\text { Low cost } \\
\text { Adjustment possibilities }\end{array}$ & $\begin{array}{l}\text { Several repetitions } \\
\text { Properties may vary } \\
\text { Sensitive ambient humidity }\end{array}$ \\
\hline Corona electrostatic & $\begin{array}{l}\text { Several containers } \\
\text { Accuracy }\end{array}$ & $\begin{array}{l}\text { Small particle size } \\
\text { Shape effect } \\
\text { A lot of parameters }\end{array}$ \\
\hline NIR & $\begin{array}{l}\text { Rapid and reliable identification } \\
\text { Low environmental impact }\end{array}$ & $\begin{array}{l}\text { Cost } \\
\text { Dark materials } \\
\text { Treatment effect (e.g. coating) }\end{array}$ \\
\hline$X$-ray & $\begin{array}{l}\text { No color problems } \\
\text { High productivity }\end{array}$ & $\begin{array}{l}\text { Overheating } \rightarrow \text { destroys material / need } \\
\text { for cooling }\end{array}$ \\
\hline
\end{tabular}

The term "low cost" is mentioned in connection with many technologies, and this may be slightly controversial, because the cost of technologies has not been evaluated between them, but the term is based on information presented in the references. Therefore, reliable cost analysis needs to be done to compare the technologies with the same standard.
Selecting the best sorting method may produce major challenges, and the measurement technique for comparison has a significant effect. If the comparison is carried out from the economical viewpoint, then for example manual, sinkfloat, and optical sorting will not necessarily turn out to be the prime choice. The apparatus of optical sorting is costly, and the use of manual and sink-float sorting methods causes extra costs (labor cost and waste disposal fee). One factor in 
the selection of the method is its ability to identify various materials. For this reason, magnetic separation appears in a negative light, while optical methods are positive. The methods that need some pre or post-treatment are in a weaker position compared to the methods that can operate independently. An important feature that will influence sorting efficiency is the size of the fraction, so treatment before sorting must be taken into account in the planning. The significance of fraction size will be emphasized in the case of electric conductivity based sorting, where the effective fraction size varies from below $1 \mathrm{~mm}$ to over 10 $\mathrm{mm}$, depending on the method. The electric conductivity based sorting methods may be excellent methods in e-waste sorting objects, for example. In addition to the size, the properties of the materials have a great effect on the sorting ability. The significance of materials will be emphasized when dealing with objects, which consist of several materials, as for example a plastic film may be layered of a mixture of various plastics.

Based on the above findings and discussion, it is now possible to suggest that an effective sorting system may consist of several sorting methods and a combination of them. In the future, would be useful to investigate which kind of combination methods will achieve the best possible sorting result, and especially a minor amount of treatment steps could be favorable. However, the suitability of each method must be evaluated case by case. Even though the method were presented negatively, it may be a suitable method for a certain target. For example, sink-float sorting is restricted in many applications due to its water disposal concerns, but it may be a possible sorting method for hospital waste. The mixed waste of a hospital in the Helsinki metropolitan area includes $26 \%$ of plastic waste [105], and for these, the sink-float method may be a useful technology, combined with a medium that performs disinfection of materials simultaneously with sorting.

As stated in the introduction section, the European Commission has made a legislative proposal to increase recycling of municipal and packaging waste to $65 \%$ and $80 \%$ by 2030 . Insofar as the legislative proposal with regard to recycling by 2030 will come true, it will have a significant impact on waste management, and especially on waste sorting. Even though the influence will be visible first in Europe, it can be possible and even probable that the decree has a global effect later on.

The opportunities to increase the recycling rate can be found in mixed waste. For example, in a survey in the Helsinki metropolitan area in 2015 it was found that the mixed waste of households contained $17 \%$ fiber materials, including paper, board, cardboard, and wood. Correspondingly, the household mixed waste included $16 \%$ of plastic waste [105]. In addition to the opportunities, mixed waste sorting may include challenges, because mixed waste can contain several sorts of waste fractions. Small steps towards to MSW recycling have been taken, as for example $41 \%$ of MSW was recycled in Finland in 2015 [106], and this value had increased by eight percentage points compared to the previous year [107]. However, in order to achieve the recycling targets set by the EU, mixed waste is the fraction which must be sorted and recycled and this topic is will be an actual theme in the following years.

\section{Conclusion}

An important issue in the world is minimizing the amount of waste, and its utilization is a solution that makes sorting an important process in the field of recycling. In this study, several treatment and sorting methods were studied and discussed.

Most of the sorting methods have been in use in mineral processing for decades. However, the waste sorting technology takes its first steps with only a few years' experience. The studied technologies showed that productivity can be quite high, but it depends on the parameters used, such as the feeding speed and similar particle size. Based on this study, for example electric conductivity based sorting and optical sorting can be potential sorting technologies, as these methods are based on the material's own properties and they do not need any extra substances that may be a problem afterwards. A technology may be quite sensitive to a feature, such as a certain color. The recoverability and efficiency of technologies, such as how much material is separated with each technique, is a potential certain topic that must be discussed in further studies. In addition, efficient separation may require several repetitions for the results of sorting to be sufficiently high and a combination of methods may be necessary. It can be concluded that many studies on waste sorting technologies will be needed in the future to achieve efficient sorting results.

\section{Acknowledgements}

This research was supported by a grant from the CIRCWASTE-FINLAND (LIFE15 IPE/FI/004) research program, coordinated by the Finnish Environment Institute (SYKE).

This is an Open Access article distributed under the terms of the Creative Commons Attribution License

\section{References}

1. Y. C. Moh and L. A. Manaf, Resour. Conserv. Recy. 116, 1 (2017).

2. D. G. Zhang, S. K. Tank and R. M. Gersberg. J. Environ. Manage. 91, 1623 (2010).

3. M. Sadat-Shojai and G-R. Bakhshandeh, Polym. Degrad. Stabil. 96, 404 (2011).

4. http://scp.eionet.europa.eu/publications/WP2012_1/wp/WP2012_1 (accessed on 30 October 2017)

5. http://www.finlex.fi/fi/laki/alkup/2010/20101126 (accessed on 16 December 2016)

6. http://www.finlex.fi/fi/laki/alkup/2015/20151401. (accessed on 16 December 2016)
7. http://eur-lex.europa.eu/legal-content/EN/TXT/PDF/?uri=CELEX:52017DC0490\&from=EN (accessed on 30 October 2017)

8. http://eur-lex.europa.eu/legalcontent/EN/TXT/?uri=celex\%3A32008L0098 (accessed on 27 November 2017)

9. http://eur-lex.europa.eu/resource.html?uri=cellar:e669092f-01e111e4-831f-01aa75ed71a1.0001.01/DOC_1\&format=PDF (accessed on 7 August 2017)

10. http://eur-lex.europa.eu/resource.html?uri=cellar:c2b5929d-999e11e5-b3b7-01aa75ed71a1.0018.02/DOC_1\&format=PDF (accessed on 18 September 2017) 
11. F. Bezati, D. Froelich, V. Massardier and E. Maris, Resour. Conserv. Recy. 55, 1214 (2011).

12. W-L. Huang, D-H. Lin, N-B Chang and K-S, Lin, Resour. Conserv. Recy. 37, 23 (2002).

13. C. Cimpan, A. Maul, M. Jansen, T. Pretz and H. Wenzel, J. Environ. Manage. 156, 181 (2015).

14. C. Cimpan, A. Maul, H. Wenzel and T. Pretz, J. Clean. Prod. 112, 4387 (2016)

15. C-Q. Wang, W. Hui, J-G. Fu and Y-N. Liu, Waste Manage. 41, 28 (2015)

16. L. Gu and T. Ozbakkaloglu, Waste Manage. 51, 19 (2016)

17. M. R. Gent, M. Menendez, J. Toraño and I. Diego, Waste Manage. Res. 27, 175 (2009)

18. D. Briassoulis, M. Hiskakis and E. Babou, Waste Manage. 33, 1516 (2013)

19. S. K. Najafi, Waste Manage. 33, 1898 (2013)

20. K. Hamad, M. Kaseem and F. Deri, Polym. Degrad. Stabil. 98, 2801 (2013)

21. N. Petchwattana, S. Covavisaruch and J. Sanetuntikul, Constr. Build. Mater. 28, 557 (2012)

22. S. Ponstabodee, N. Kunachitpimol and S. Damronglerd, Waste Manage. 28, 475 (2008)

23. N. T. T. Truc and B-K. Lee, Environ. Sci. Technol. 50, 10580 (2016)

24. G. Richard, S. Touhami, T. Zeghloul and L. Dascalescu, Waste Manage. 60, 112 (2017)

25. https://i.unu.edu/media/unu.edu/news/52624/UNU-1stGlobal-EWaste-Monitor-2014-small.pdf (accessed on 8 August 2017)

26. K. Huang, J. Guo and Z. Xu, J. Hazard. Mater. 164, 399 (2009)

27. P. Hadi, M. Xu, C. S. K. Lin, C-W. Hui and G. McKay, J. Hazard. Mater. 283, 234 (2015)

28. A. Chagnes, G. Cote, C. Ekberg, M. Nilsson and Retegan, WEEE Recycling. Research, Development, and Policies, Elsevier, Amsterdam (2016)

29. M. Burke, Chem. World UK. 4, 45 (2007)

30. B. M. Caballero, I. de Marco, A. Adrados, A. LópezUrionabarrenechea, J. Solar and N. Gastelu, Waste Manage. 57, 226 (2016)

31. S. Salhofer, B. Steuer, R. Ramusch and P. Beigl, Waste Manage. 57, 27 (2016)

32. K. Parajuly and H. Wenzel, J. Clean. Prod. 151, 272 (2017)

33. K. Parajuly, K. Habib and G. Liu, Resour. Conserv. Recy. 123, 85 (2017)

34. J. Bachér and T. Kaartinen, Waste Manage. 60, 609 (2017)

35. J. Cui and E. Forssberg, J. Hazard. Mater. B99, 243 (2003)

36. E. Worrell and M. A. Reuter, Handbook of recycling: state-of-theart for practitioners, analysts, and scientists, Elsevier, Waltham (2014)

37. A. Mwanga, M. Parian, P. Lamberg and J. Rosenkranz, Miner, Eng. 111, 182 (2017)

38. C. Xuan, L. Cao, P. Wu, Y. Ma and D. Han, Telkomnika. 10, 1151 (2012)

39. S. Naik and B. Chaudhuri, WIT Trans Model Sim. 51, 121 (2011)

40. L. J. Naimi, F. Collard, X. Bi, J. Lim and S. Sokhansanj, Biomass. Conv. Bioref. 6, 397 (2016)

41. M. R. Gent, M. Menendez, J. Toraño and S, Torno, Resour. Conserv. Recy. 55, 472 (2011)

42. E. Grant and H. Kalman, Adv. Powder. Technol. 13, 233 (2002)

43. G. Tchobanoglous and F. Kreith, Handbook of solid waste management, The McGraw-Hill Companies Inc. (2002)

44. H. Yuan, S. Fu, W. Tan, J. He and K. Wu, Waste Manage. 45, 108 (2015)

45. http://sciencenordic.com/machines-are-better-people-sortinghousehold-trash (accessed on 27 January 2017)

46. K. Zhang, Y. Wu, W. Wang, B. Li, Y. Zhang and T. Zuo, Resour. Conserv. Recy. 104, 276 (2015)

47. Y-S. Chen, S-S. Hsiau, H-Y. Lee, Y-P. Chyou and C-J. Hsu, Chem. Eng. Process. 49, 1214 (2010)

48. S. T. Lau, W. H. Cheung, C. K. Kwong, C. P. Wan, K. K. H. Choy, C. C. Leung C C et al. Waste Manage. 25, 1004 (2005)

49. http://ec.europa.eu/environment/life/project/Projects/index.cfm?fus eaction $=$ home.showFile\&rep=file\&fil=PLASTIC_ZERO_annex_d 32_action4.2_report_on_assessment_sept2013_final.pdf (accessed on 27 March 2017 )

50. J. Kortnik and J. Leskovar, RMZ - M\&G. 60, 143 (2013)

51. M. Jansen, E. U. T. van Velzen and T. Pretz, Handbook for the sorting of plastic packaging waste concentrates, Wageningen UR Food \& Biobased Reasearch (2015)

52. J. Ruan and Z. Xu, Waste Manage. 31, 2319 (2011)
53. B. Hu, S. Serranti, N. Fraunholcz, F. Di Maio and G. Bonifazi, Waste Manage. 33, 574 (2013)

54. V. Luciani, G. Bonifazi, P. Rem and S. Serranti, Waste Manage. 45, 118 (2015)

55. S. Serranti, V. Luciani, G. Bonifazi, B. Hu and P. C. Rem, Waste Manage. 35, 12 (2015)

56. E. J. Bakker, P. C. Rem and N. Fraunholcz, Waste Manage. 29, 1712 (2009)

57. E. Lupo, M. Moroni, F. La Marca, S. Fulco and V. Pinz, Waste Manage. 51, 3 (2016)

58. F. Pita and A. Castilho, Waste Manage. 28, 89 (2016)

59. G. I. Schwerzler, Powder Technol. 160, 135 (2005)

60. R. D. Pascoe, Waste Manage. 26, 1126 (2006)

61. C-H. Park, H-S. Jeon, H-S. Yu, O-H. Han and J-K. Park, Environ. Sci. Technol. 42, 249 (2008)

62. G. D. Dodbiba, N. Haruki, A. Shibayama, T. Miyazaki and T. Fujita, Int. J. Miner. Process. 65, 11 (2002)

63. G. Dodbiba, J. Sadaki, K. Okaya, A. Shibayama and T. Fujita, Miner. Eng. 18, 1350 (2005)

64. G. Dodbiba, A. Shibayama, J. Sadaki and T. Fujita, Mater T JIM. 44, 2427 (2003)

65. M. T. Carvalho, E. Agante and F. Durão, Waste Manage. 27, 1747 (2007)

66. H. Shent, R. J. Pugh and E. Forssberg, Resour. Conserv. Recy. 25, 85 (1999)

67. S. Saisinchai, Eng J-Canada. 18, 45 (2014)

68. C-Q. Wang, H. Wang, B-X. Wu and Q. Liu, Waste Manage. 34, $1206(2014)$

69. C. Wang, H. Wang, J. Fu, L. Zhang, C. Luo and Y. Liu, Waste Manage. 45, 112 (2015)

70. S. R. Mallampati, J. H. Heo and M. H. Park, J. Hazard. Mater. 306, 13 (2016)

71. M. Censori, F. La Marca and M. T. Carvalho, Waste Manage. 54, 39 (2016)

72. M. Amir, M. Karim, B. B. Mourad and T. Amar, Int. J. Environ. Stud. 73, 203 (2016)

73. D. Wang, X, Ma, X, Zhi and S. Zhang, Sensors \& Transducers. 158, 242 (2013)

74. J. Ruan, L. Dong, J. Zheng, T. Zhang, M. Huang and Z. Xu, Waste Manage. 60, 84 (2017)

75. J. Ruan and Z. Xu, J. Hazard. Mater. 192, 307 (2011)

76. Z. Schlett and M. Lungu, Miner. Eng. 15, 365 (2002)

77. J. Li, Y. Jiang and Z. Xu, J. Clean. Prod. 141, 1316 (2017)

78. C-H. Park, H-S. Jeon and J-K. Park, J. Hazard. Mater. 144, 470 (2007)

79. G. Wu, J. Li and Z. Xu, Waste Manage. 33, 585 (2013)

80. A. Younes, M. Younes, H. Sayah, A. Samuila and L. Dascalescu, Particulate Sci. Technol. 33, 189 (2015)

81. J-K. Lee and J-H. Shin, Korean J. Chem. Eng, 19, 267 (2002)

82. S. Bendimerad, A. Tilmatine, M. Ziane and L. Dascalescu, Int. J. Environ. Stud. 66, 529 (2009)

83. J. Wu, J. Li and Z. Xu, J. Hazard. Mater. 159, 230 (2008)

84. A. Tilmatine, K. Medles, S-E. Bendimerad, F. Boukholda and L. Dascalescu, Waste Manage. 29, 228 (2009)

85. T. Zeghloul, L. Dascalescu, S. Touhami, M. Miloudi, O. Dahou and G. Richard. Industry Applications Society Annual Meeting, Addison, USA, (2015)

86. J. Li, H. Lu, J. Guo, Z. Xu and Y. Zhou, Environ. Sci. Technol. 41, 1995 (2007)

87. J. Li, Q. Zhou and Z. Xu, Waste Manage. Res. 32, 1227 (2014)

88. G. Bedeković, B. Salopek and I. Sobota, Technical Gazette. 18, 261 (2011)

89. J. Li, H. Lu, Z. Xu and Y. Zhou, J. Hazard. Mater. 154, 331 (2008)

90. J. Li, H. Lu, S. Liu and Z. Xu, J. Hazard. Mater. 153, 269 (2008)

91. P, Tatzer, M. Wolf and T. Panner, Real-Time Imaging. 11, 99 (2005)

92. M. T. Carvalho, C. Ferreira, A. Portela and J. T. Santos, Waste Manage. 29, 1138 (2009)

93. I. Vegas, K. Broos, P. Nielsen, O. Lambertz and A. Lisbona, Constr. Build. Mater. 75, 121 (2015)

94. C-L. So, S. T. Lebow, L. H. Groom and T. G. Rials, Wood Fiber. Sci. 36, 329 (2004)

95. S. Serranti, A. Gargiulo and G. Bonifazi, Resour. Conserv. Recy. 61, 52 (2012)

96. S. Lotfi, F. Di Maio, H. Xia, S. Serranti, R. Palmieri and G. Bonifazi, Proceedings of the 15th Euroseminar on Microscopy Applied to Building Materials, Delft (2015)

97. A. Feil, T. Pretz, M. Jansen and E. U. T. van Velzen, Waste Manage, Res. 35, 172 (2017) 
98. C. N. Block, T. Shibata, H. M. Solo-Gabriele and T. G. Townsend, Environ. Pollut. 148, 627 (2007)

99. F. Bezati, D. Froelich, V. Massardier and E. Maris, Waste Manage. 30, $591(2010)$

100.A. R. Hasan, J. Schindler, H. M. Solo-Gabriele and T. G. Townsend, Waste Manage. 31, 688 (2011)

101.A. R. Hasan, H. Solo-Gabriele and T. Townsend, Waste Manage. 31, 695 (2011)

102.A. Aldrian, A. Ledersteger and R. Pomberger, Waste Manage. 36, 297 (2015)

103.S. Serranti, A. Gargiulo and G. Bonifazi, Waste Manage. 31, 2217 (2011)
104.M. A. Gondal and M. N. Siddiqui, J. Environ. Sci. Heal. A. 42, 1989 (2007)

105.https://www.hsy.fi/sites/Esitteet/EsitteetKatalogi/Raportit/Paakaupu nkiseudun_seka-ja_biojatteen_koostumus_vuonna_2015.pdf (accessed on 29 March 2017)

106.http://www.stat.fi/til/jate/2015/jate 2015 2016-12-

20 tie_001_en.html (accessed on 28 March 2017)

107.http://www.stat.fi/til/jate/2014/jate_2014_2015-12-

01 tie 001 en.html (accessed on 28 March 2017)

108.C-Q. Wang, H. Wang amd Y-N. Liu, Waste Manage. 35, 42 (2015) 